\title{
Facial Venous Malformations Are Associated with Cerebral Developmental Venous Anomalies
}

\author{
(D)W. Brinjikji, (D) C.A. Hilditch, (D)A.C. Tsang, (DP.J. Nicholson, (D). Krings, and (D). Agid
}

\begin{abstract}
BACKGROUND AND PURPOSE: A number of studies have demonstrated the existence of segmental vascular disorders affecting soft tissues of the head and neck along with the intracranial vasculature. The purpose of this study was to determine whether there is an association between cerebral developmental venous anomalies and venous malformations of the face, head, and neck.
\end{abstract}

MATERIALS AND METHODS: A consecutive series of patients with head and neck venous malformations who underwent MR imaging of the brain with postcontrast $\mathrm{T1}$ - or $\mathrm{T} 2^{*}$-weighted imaging were included. Developmental venous anomaly prevalence in this patient population was compared with an age- and sex-matched control group without venous malformations at a ratio of 1:2. All images were interpreted by 2 neuroradiologists. Data were collected on venous malformation location, developmental venous anomaly location, developmental venous anomaly drainage pattern, and metameric location of venous malformations and developmental venous anomalies. Categoric variables were compared using $\chi^{2}$ tests.

RESULTS: Forty-two patients with venous malformations were included. The mean age was $38.1 \pm 11.1$ years, and $78.6 \%$ of patients were female. The prevalence of developmental venous anomalies in this patient population was $28.6 \%$. The control population of 84 patients had a mean age of $40.0 \pm 5.9$ years, and $78.6 \%$ of patients were female. The prevalence of developmental venous anomalies in this patient population was $9.5 \%(P=.01)$. In $83.3 \%$ of cases, developmental venous anomalies were ipsilateral to the venous malformation, and in $75 \%$ of cases, they involved the same metamere.

CONCLUSIONS: Our case-control study demonstrated a significant association between brain developmental venous anomalies and superficial venous malformations. These findings suggest that there may be a similar pathophysiologic origin for these 2 entities.

ABBREVIATIONS: CVMS = Cerebrofacial venous metameric syndrome; DVA $=$ developmental venous anomaly; $V M=$ venous malformation

W ith increased use of cross-sectional imaging, developmental venous anomalies (DVAs) are discovered more frequently. DVAs consist of dilated intramedullary veins that converge into a larger draining vein, which then drains into either the superficial or deep venous system. Population-based studies have found that up to $10 \%$ of the general population has incidental DVAs. ${ }^{1}$

While DVAs are generally common, superficial venous vascular malformations of the head and neck are quite rare. Venous malformations (VMs) are slow-flow vascular malformations that manifest as soft-tissue swellings under normal or bluish skin.

\footnotetext{
Received June 30, 2018; accepted after revision August 6.
}

From the Departments of Radiology (W.B.) and Neurosurgery (W.B.), Mayo Clinic, Rochester, Minnesota; and Joint Department of Medical Imaging (W.B., C.A.H., A.C.T., P.J.N., T.K., R.A.), Toronto Western Hospital, Toronto, Ontario, Canada. Please address correspondence to Waleed Brinjikji, MD, Mayo Clinic, 200 1st St SW, Rochester, MN 55905; e-mail: brinjikji.waleed@mayo.edu; @wbrinjikji

三 Indicates article with supplemental on-line table.

http://dx.doi.org/10.3174/ajnr.A5811
These lesions are exacerbated by increased central venous pressure such as a Valsalva maneuver. Pathologically, VMs consist of dilated venous channels in the dermis or muscular tissues that usually drain into larger tributaries of the external jugular venous system. $^{2}$

During the past several years, we have noticed a possible association between the presence of VMs and intracranial DVAs on cross-sectional imaging. This may be a logical association given that the presence of metameric disorders in which patients have vascular malformations involving the brain and soft tissues of the head, face, and neck is well-established. ${ }^{3}$ Identification of such an association could also be important because it could provide some insight into the pathogenesis of both of these entities. In order to study the association between DVAs and VMs, we performed a casecontrol study examining the prevalence of DVAs on MR imaging of a consecutive population of patients with VMs compared to a group of age- and sex-matched controls. 


\section{MATERIALS AND METHODS}

\section{Patient Population}

Following institutional review board approval at Toronto Western Hospital, we queried our data base of $>250$ patients with VMs for patients who had an MR imaging of the brain including either T2* -weighted imaging and/or postcontrast T1-weighted imaging. VMs were confirmed by a combination of physical examination and imaging-based findings. ${ }^{4,5}$ Imaging criteria for a VM on MR imaging were the following: 1) a septate lobulated T2 hyperintense and T1 hypointense mass without mass effect; 2) phleboliths, which are characteristically hypointense on T1/T2; 3) the presence of fluid-fluid levels; 4) no flow voids on spin-echo sequences; 5) the lesion infiltrating tissue planes; 6) no arterial or early venous enhancement; and 7) diffuse enhancement on delayed images. On clinical examination, VMs appear as faint blue, soft, and easily compressible nonpulsatile masses. The lesions characteristically enlarge with a Valsalva maneuver and in dependent positions and decompress with local compression. Only adult patients with VMs were included in this study because our institution is not a pediatric center. We also selected a group of age- and sex-matched controls (case:control ratio of 1:2) from a data base used to estimate the prevalence of brain DVAs in the general population, which was reviewed by 2 board-certified neuroradiologists. Age matching was performed with an error margin of \pm 1 year. Thus, a 37-year-old woman could be matched with a 36- to 38-year-old woman.

\section{Imaging Analysis}

For the VM population, all images were analyzed by 2 neuroradiologists. Images were reviewed to document the following: 1) the presence or absence of a DVA, 2) the location and side of the DVA if present, and 3 ) the location and side of the VM. Locations were categorized by metamere as well. The 3 metameres of the craniofacial system include the medial prosencephalic group (olfactory) with involvement of the forehead, nose hypothalamus, corpus callosum, and hypophysis (cerebrofacial venous metameric sydrome [CVMS] 1); the lateral prosencephalic group (optic) with involvement of the temporoparieto-occipital lobes, optic nerve, retina, thalamus, eye, cheek, and maxilla (CVMS 2); and the rhombencephalic/mesencephalon (otic) group with involvement of the cerebellum, brain stem, lower face, mandible, petrous bone, and maxilla (CVMS 3). ${ }^{6}$

\section{Statistical Analysis}

The primary outcome of this study was the prevalence of DVAs in the VM population and in controls. Prevalence rates were compared using a $\chi^{2}$ test. A Student $t$ test was used to compare continuous variables. All statistical analyses were performed using JMP 13.0 (SAS Institute, Cary, North Carolina).

\section{RESULTS}

\section{Patient Population}

Forty-two patients with VMs were included along with 84 controls. In the VM population, the mean age was $38.1 \pm 11.1$ years, and $78.6 \%$ of patients were women (33/42). The control population had a mean age of $40.0 \pm 5.9$ years, and $78.6 \%$ of patients were women (66/84). Among the patients with VMs, 3 patients had bilateral VMs and 39 patients had unilateral facial VMs. Of the patients in the control group, 11 patients underwent MR imaging for evaluation of seizure, 7 patients underwent MR imaging for evaluation of an intracranial mass, and 66 patients underwent MR imaging for other causes, including headache, metastatic disease screening, and other indications such as ruling out ischemia.

\section{DVA Prevalence and Characteristics}

Of the 42 patients with VMs, a diagnosis of DVA was made in 12 patients $(28.6 \%)$. The prevalence of DVAs in the control population was $9.5 \%(8 / 84)$. This difference was statistically significant $(P=.01)$.

Of the patients with VMs and DVAs, none were men and 12 were women. All 12 had extensive cutaneous VMs. DVAs were unilateral in 8 patients and bilateral in 4 patients. There were 35 DVAs, with 7 patients having multiple DVAs. Thirty DVAs had deep drainage, and 5 had superficial venous drainage. In 23 cases, DVAs were supratentorial, and in 12 cases, they were infratentorial. In $83.3 \%$ of cases, DVAs were ipsilateral to the $\mathrm{VM}$, and in $75 \%$ of cases, they involved the same metamere. The On-line Table summarizes the characteristics of all patients with VMs with associated DVAs. Case examples are provided in Figs 1-3.

\section{DISCUSSION}

Our case-control study examining the prevalence of DVAs in patients with VMs and a group of control patients demonstrated a number of interesting findings. First, the prevalence of DVAs in the VM population was $>2$ times higher than that in the general population. Most interesting, more often than not, DVAs were located along the same metamere and/or side as the VM. These findings are important because they suggest that there may be a similar pathophysiologic or embryologic basis to both craniofacial VMs and intracranial DVAs.

Prior studies have demonstrated a possible association between DVAs and VMs. However, no case-control studies have been performed to date comparing the prevalence of DVAs in patients with VMs and in a group of patient controls without VMs. In a series of 40 patients who underwent cerebral angiography as part of the evaluation of facial venous vascular malformations, Boukobza et $\mathrm{al}^{7}$ identified 8 patients (20\%) with complex DVAs, with most patients having multiple DVAs. Unlike SturgeWeber Syndrome, these complex DVAs were associated with a normal superficial cortical venous system. Most DVAs were supratentorial and had large flaming venous radicles that drained into a tortuous deep venous system, and most were ipsilateral to the facial VM. One patient had a symptomatic cavernoma that required resection. ${ }^{7}$ Overall, the DVA prevalence rate and clinical presentation of these VM-associated DVAs are very similar to those seen in our patient population. A number of additional case reports have been published concerning patients with extensive facial venous malformations and associated ipsilateral DVAs. Such an association hardly appears to be coincidental. ${ }^{2,3,6,8-10}$

DVAs are thought to form in later periods of cerebral venous development as functional adaptations to thrombosis or failure 


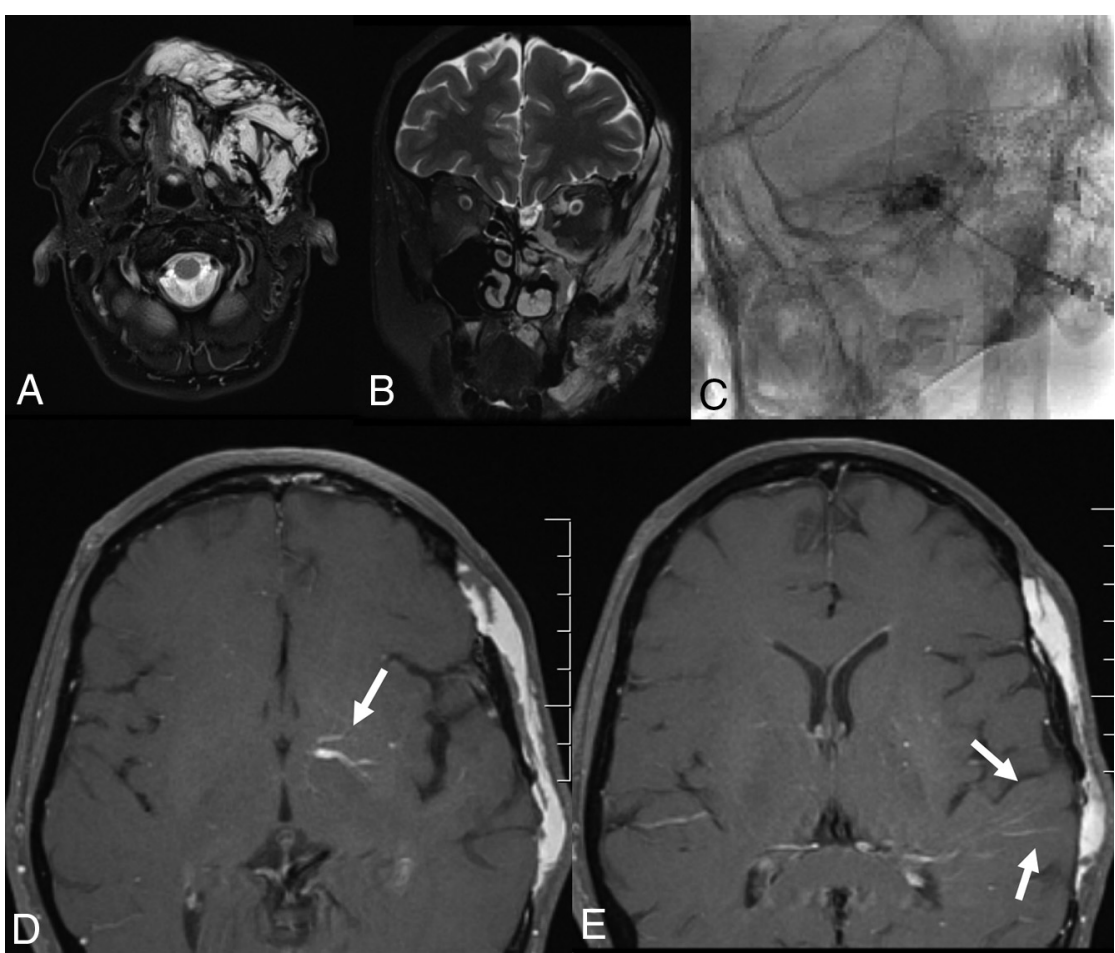

FIG 1. A 49-year-old woman with VMs and DVAs. VMs are in the left temporal region, orbit, zygomaticofacial region, and mandible $(A$ and $B)$. The patient underwent bleomycin sclerotherapy for treatment of the VMs with good results $(C)$. She also had an extensive DVA of the left temporal lobe, basal ganglia, and left cerebellar hemisphere ( $D$ and $E)$. Findings would be consistent with CVMS 1-3.

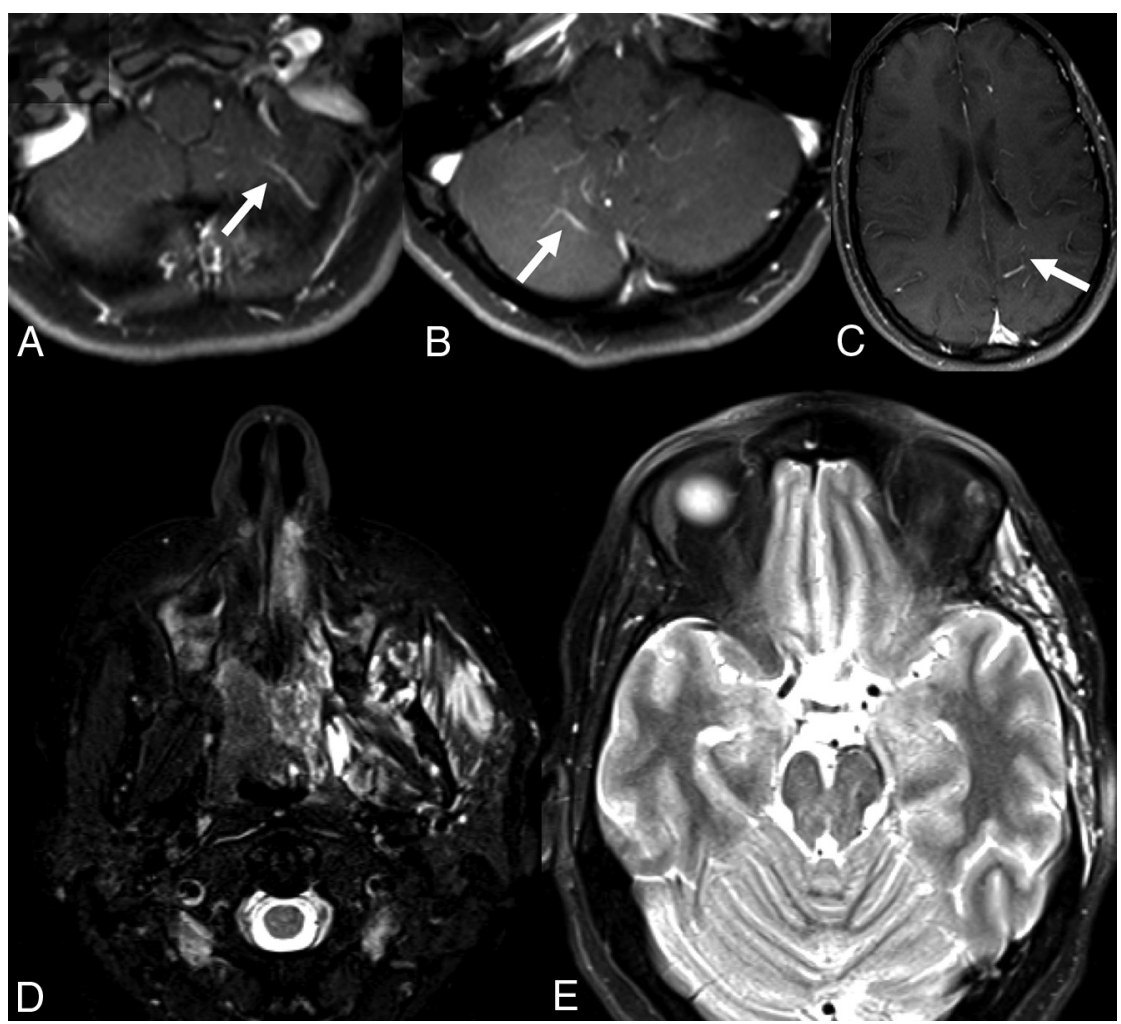

FIG 2. A 30-year-old man with facial VMs and left supratentorial DVAs. A-C, Postcontrast T7weighted MRIs show DVAs in the bilateral cerebellar hemispheres, left parietal lobe, and left temporal lobe. $D$ and $E, T 2$-weighted MR imaging shows extensive VMs of the left zygomaticotemporal region and masticator spaces. of development of superficial or deep veins. ${ }^{11}$ It is possible that early postnatal venous occlusion could trigger remodeling of medullary veins, thus resulting in DVA formation. Furthermore, when DVAs become symptomatic, it is invariably due to thrombosis of the collector vein or a venous radicle. ${ }^{11}$ Nonetheless, the common final pathway is an error in vascular embryogenesis resulting in occlusion and maldevelopment of normal venous structures in a given part of the brain. To date, there have been no genetic mutations associated with DVA development.

Some authors have postulated that a similar error in vascular embryogenesis (ie, thrombosis resulting in occlusion and maldevelopment) affecting the craniofacial venous vasculature could result in the formation of VMs as well. Like DVAs, VMs generally do not develop de novo or proliferate and spread to other vascular beds in adult life. Most interesting, some authors have suggested that VMs are also thought to form due to a procoagulable state. ${ }^{12,13}$ In a case-control study of patients with and without VMs, Dompmartin et $\mathrm{al}^{12,13}$ found that $43 \%$ of patients with VMs have an elevated D-dimer level compared with just $4 \%$ of patients without VMs. Up to one-third of children with VMs have some form of prothrombotic coagulopathy. ${ }^{14}$ In another study, Dompmartin et $\mathrm{al}^{12,13}$ found that almost $50 \%$ of patients with VMs have local intravascular coagulation, a factor thought to be responsible for VM enlargement and pain. So how can we explain the link between DVAs and extensive facial VMs in a more or less unilateral distribution in this patient population? Given that both VMs and DVAs are associated with some degree of prothrombotic state, we hypothesize that VMs and DVAs may develop due to an increased predilection for local venous thrombosis and occlusion, possibly due to a metameric disorder related to venous endothelial dysfunction.

Our study has both practical implications and implications for future research. First, on the basis of these findings, one might consider a whole-head MR imaging for patients with VMs to evaluate intracranial vascular abnormalities. Two patients had DVA-associated cavernomas, one of which developed de novo and was associated with clinical 


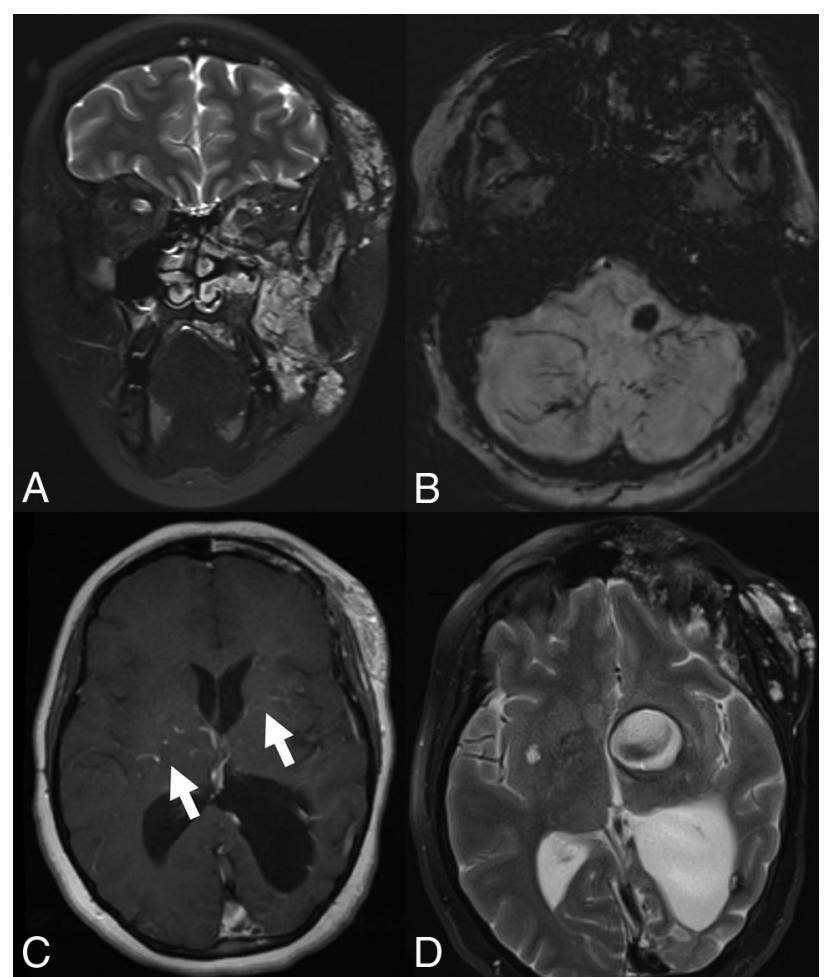

FIG 3. A 22-year-old woman with a left facial DVA, bilateral VMs, and a de novo cavernoma. A, Coronal T2-weighted MR imaging demonstrates a vascular malformation involving the soft tissues overlying the left zygomatico-orbital region with extension to the left maxillary region. $B$, Axial SWI MR imaging of the brain demonstrates extensive DVAs of the bilateral cerebellar hemispheres and a cavernoma of the left medulla. C, Postcontrast MR imaging demonstrates venous radicles of 2 DVAs involving the bilateral basal ganglia. $D, T 2$-weighted MR imaging 2 years later demonstrates a large cavernoma that developed in one of the venous radicles of the left basal ganglia DVA.

symptoms. Identification of extensive DVAs in the patient population with VMs could be used to select patients who may require closer imaging surveillance. Regarding implications for future research, the genetic and pathophysiologic mechanisms that result in the codevelopment of DVAs and VMs should be further studied. Three patients in our study had either a classic Sturge-Weber syndrome or a form fruste of Sturge-Weber. It is now well-established that Sturge-Weber is due to a somatic mutation in the GNAQ gene, which plays a role in expression of the endothelin in vascular endothelial cells. ${ }^{15}$ Meanwhile, somatic mutations in vascular endothelial cells of the PIK3CA gene are associated with the development of venous vascular malformations. ${ }^{16}$ Discovery of these genetic associations has led to promising research in targeted therapy for these diseases. It may be that patients with DVAs and head and neck VMs carry a common vascular endothelial cell somatic mutation. Further research into identifying genetic mutations in this patient population may provide insight into disease pathophysiology and targeted treatment of venous vascular malformations.

\section{Limitations}

Our study has limitations. First, this was a retrospective study and is prone to selection bias. It is possible that the prevalence of DVAs in both the VM and control groups is artificially high due to variations in the indications for imaging. Routine intracranial T2* and postcontrast imaging are no longer performed at our institution for evaluation of patients with VMs, and often, these imaging studies are performed for evaluation of orbital vascular malformations or in patients with VMs with neurologic symptoms such as headaches. Another limitation is that while all patients in the control group underwent a postcontrast T1-weighted sequence, this was not the case in our patient population with VMs. Postcontrast T1-weighted imaging is more sensitive than $\mathrm{T}^{*}$ weighted imaging for the detection of smaller DVAs. Thus, we could have underestimated the DVA prevalence in the VM population. On the other hand, it is possible that our study is prone to selection bias because only a subset of patients presented with brain MR imaging. Last, our study is small, including only 42 patients with VMs.

\section{CONCLUSIONS}

Our case-control study demonstrated a significant association between DVAs and superficial VMs. More often than not, DVAs were located along a similar metamere or side as the VMs. These findings suggest that there may be a similar pathophysiologic origin for these 2 entities.

\section{REFERENCES}

1. Brinjikji W, El-Masri AE, Wald JT, et al. Prevalence of cerebral cavernous malformations associated with developmental venous anomalies increases with age. Childs Nerv Syst 2017;33:1539-43 CrossRef Medline

2. Agid R, Terbrugge KG. Cerebrofacial venous metameric syndrome 2 plus 3: facial and cerebral manifestations. Interv Neuroradiol 2007; 13:55-58 CrossRef Medline

3. Krings T, Geibprasert S, Luo CB, et al. Segmental neurovascular syndromes in children. Neuroimaging Clin N Am 2007;17:245-58 CrossRef Medline

4. Flors L, Leiva-Salinas C, Maged IM, et al. MR imaging of soft-tissue vascular malformations: diagnosis, classification, and therapy follow-up. Radiographics 2011;31:1321-40; discussion 1340-41 CrossRef Medline

5. Wassef M, Blei F, Adams D, et al; VA Board and Scientific Committee. Vascular anomalies classification: recommendations from the International Society for the Study of Vascular Anomalies. Pediatrics 2015;136:e203-14 CrossRef Medline

6. Bhattacharya JJ, Luo CB, Suh DC, et al. Wyburn-Mason or BonnetDechaume-Blanc as Cerebrofacial Arteriovenous Metameric Syndromes (CAMS): a new concept and a new classification. Interv Neuroradiol 2001;7:5-17 CrossRef Medline

7. Boukobza M, Enjolras O, Guichard JP, et al. Cerebral developmental venous anomalies associated with head and neck venous malformations. AJNR Am J Neuroradiol 1996;17:987-94 Medline

8. Goulao A, Alvarez H, Garcia Monaco R, et al. Venous anomalies and abnormalities of the posterior fossa. Neuroradiology 1990;31: 476-82 CrossRef Medline

9. Portilla P, Husson B, Lasjaunias P, et al. Sturge-Weber disease with repercussion on the prenatal development of the cerebral hemisphere. AJNR Am J Neuroradiol 2002;23:490-92 Medline

10. Ramli N, Sachet M, Bao C, et al. Cerebrofacial venous metameric syndrome (CVMS) 3: Sturge-Weber syndrome with bilateral lymphatic/venous malformations of the mandible. Neuroradiology 2003;45:687-90 CrossRef Medline

11. Pereira VM, Geibprasert S, Krings T, et al. Pathomechanisms of symptomatic developmental venous anomalies. Stroke 2008;39: 3201-15 CrossRef Medline

12. Dompmartin A, Acher A, Thibon P, et al. Association of localized intravascular coagulopathy with venous malformations. Arch Dermatol 2008;144:873-77 Medline 
13. Dompmartin A, Ballieux F, Thibon P, et al. Elevated D-dimer level in the differential diagnosis of venous malformations. Arch Dermatol 2009;145:1239-44 Medline

14. Hung JW, Leung MW, Liu CS, et al. Venous malformation and localized intravascular coagulopathy in children. Eur J Pediatr Surg 2017;27:181-84 CrossRef Medline
15. Shirley MD, Tang H, Gallione CJ, et al. Sturge-Weber syndrome and port-wine stains caused by somatic mutation in GNAQ. $N$ Engl J Med 2013;368:1971-79 CrossRef Medline

16. Limaye N, Kangas J, Mendola A, et al. Somatic activating PIK3CA mutations cause venous malformation. Am J Hum Genet 2015;97: 914-21 CrossRef Medline 